\title{
17th International Conference on Human Retroviruses: HTLV and Related Viruses, Trois llets, Martinique,(FWI). 18-21 June 2015
}

\author{
Raymond Cesaire ${ }^{1}$, Agnès Lézin ${ }^{1}$, Jean-Marie Péloponèse $\mathrm{Jr}^{2^{*}}$ \\ From 17th International Conference on Human Retroviruses: HTLV and Related Viruses \\ Trois Ilets, Martinique. 18-21 June 2015
}

\section{Introduction}

The 17th International Conference on Human Retrovirology: HTLV and Related Retroviruses took place in Trois Ilets, Martinque (FWI) from June 18th to June 21th, 2015. The main topic covered during the meeting was human T-lymphotropic viruses (HTLVs) and was subdivided in distinct sessions: clinical research, animal models, immunology, molecular and cellular biology, and virology. The following abstracts were presented.

Five of the presented abstracts are not included in this supplement as they have been published elsewhere [1-5].
Transformation. PLoS Pathog 2014, 10(10):e1004458, doi:10.1371/journal. ppat.1004458.

5. Jain P, Lavorgna A, Sehgal M, Gao L, Ginwala R, Sagar D, Harhaj EW, Khan JK: Myocyte enhancer factor (MEF)-2 plays essential roles in T-cell transformation associated with HTLV-1 infection by stabilizing complex between Tax and CREB. Retrovirology 2015, 12:23, doi:10.1186/s12977-0150140-1.

\section{doi:10.1186/1742-4690-12-S1-I1}

Cite this article as: Cesaire et al: 17th International Conference on Human Retroviruses: HTLV and Related Viruses, Trois llets, Martinique, (FWI). 18-21 June 2015. Retrovirology 2015 12(Suppl 1):11.

\section{Authors' details}

'Laboratoire de Virologie-Immunologie, EA 4537, Centre Hospitalier Universitaire de Fort-de-France, Fort-de-France, Martinique, France. ${ }^{2}$ Centre d'Études d'Agents Pathogènes et Biotechnologies pour la Santé, CNRS FRE 3689, Université Montpellier 1, Université Montpellier 2, Montpellier, France.

Published: 28 August 2015

\section{References}

1. Filippone C, Betsem E, Tortevoye P, Cassar O, Bassot S, Froment A, Fontanet $A$, Gessain A: A severe bite from a non-human primate is a major risk factor for HTLV-1 infection in hunters from Central Africa. Clin Infect Dis 2015, doi: 10.1093/cid/civ145.

2. Billioux BJ, Massoud R, Ohayon J, Cortese I, Ratner L, Caruso B, Johnson K, Alahata Y, Jacobson S: Pilot Study of Raltegravir, an Integra se Inhibitor, in HT LV-1 Associated Myelopathy/Tropical Spastic Paraparesis (HAM/TSP). J Neurovirol 2015, 21(Suppl 1), Abstract P17.

3. Anderson M, Lepene B, Ohayon J, Kasanchi F, Jacobson S: Detection of Human T-cell Lymphotropic Virus Type I proteins in exosomes from HAM/TSP patient CSF by novel nanotrap technology. J Neurovirol 2015, 21(Suppl 1), Abstract P4.

4. Choi YB, Harhaj EW: HTLV-1 Tax Stabilizes MCL-1 via TRAF6-Dependent K63-Linked Polyubiquitination to Promote Cell Survival and

\footnotetext{
* Correspondence: jean-marie.peloponese@cpbs.cnrs.fr

${ }^{2}$ Centre d'Études d'Agents Pathogènes et Biotechnologies pour la Santé, CNRS FRE 3689, Université Montpellier 1, Université Montpellier 2, Montpellier, France

Full list of author information is available at the end of the article
}

Submit your next manuscript to BioMed Central and take full advantage of:

- Convenient online submission

- Thorough peer review

- No space constraints or color figure charges

- Immediate publication on acceptance

- Inclusion in PubMed, CAS, Scopus and Google Scholar

- Research which is freely available for redistribution
() Biomed Central 